\title{
Determinants of utilization of maternity benefit schemes among mothers in urban slums of Davangere city, Karnataka, India
}

\author{
Navinkumar Angadi*, Shubha Davalgi, Raghavendra S. K.
}

Department of Community Medicine, J. J. M. Medical College, Davangere 577004, Karnataka, India

Received: 01 January 2016

Accepted: 04 February 2016

*Correspondence:

Dr. Navinkumar Angadi,

E-mail: navinkumarangadi7@gmail.com

Copyright: () the author(s), publisher and licensee Medip Academy. This is an open-access article distributed under the terms of the Creative Commons Attribution Non-Commercial License, which permits unrestricted non-commercial use, distribution, and reproduction in any medium, provided the original work is properly cited.

\begin{abstract}
Background: Maternal healthcare still a major challenge to the global public health system, especially in developing countries. Globally, every day about 830 women die from preventable causes related to pregnancy and childbirth. Many maternity benefit schemes were implemented to improve financial and geographic access to quality care for poor mothers. The objectives of the study were to 1 . Utilization of maternity benefit schemes among mothers in urban slums of davangere city. 2. Determinants of utilization of these maternity benefit schemes among mothers in urban slums. Study design was community based cross-sectional study.

Methods: This study was conducted among all the mothers who had delivered prior to one year from the start of the study in urban slums.

Results: $57 \%$ of the mothers had utilised Janani Suraksha Yojana, 29\% of mothers had utilized Prasuthi Araike Yojana, $65 \%$ of mothers had utilized Madilu Yojana and none of mothers had utilized Thayi Bhagya schemes. The major source of information was health care workers. Major reason for non-utilization of maternity benefit schemes was lack of awareness.

Conclusions: Overall the utilization of various maternity benefit schemes is poor among beneficiaries. Mothers and their husband's literacy status, occupation of mother, type of family, socioeconomic status are the important factors that affect utilization of various schemes.
\end{abstract}

Keywords: Maternity benefit schemes, Urban slums, Utilization

\section{INTRODUCTION}

Maternal healthcare still a major challenge to the global public health system, especially in developing countries. ${ }^{1}$ Globally every day about 830 women die from preventable causes related to pregnancy and childbirth and $99 \%$ of these women are from developing countries. Half of these deaths occur in sub-Saharan Africa and almost one third report from south Asia. ${ }^{2}$

Under the Millennium Development Goal (MDG) 5, the target was to reduce Maternal Mortality Ratio (MMR) by three quarters between 1990 \& 2015. Based on the UN Inter-Agency Expert Group's publication the target for MMR was 140 per 1,00,000 live births by the year 2015 .
As per Sample Registration System 2011-13, MMR in India has shown a decline from 398 per 100,000 live births (1997-98) to 167 per 100,000 live births (2001415). ${ }^{3}$ Institutional deliveries in India have increased from $38.7 \%$ (National Family Health Survey-3, 2005-06) to $46.9 \%$ (District level health survey, 2007-08). As per the coverage evaluation survey (CES 2009) conducted by the UNICEF institutional deliveries rate was $72.9 \% .^{4}$

Efforts to address the issue of high maternal and infant mortality rate through promotion of institutional deliveries gained momentum with the formulation of National Rural Health Mission (NRHM). Many schemes under NRHM were implemented to improve financial and geographic access to quality care for poor mothers 
and state governments also introduced various schemes. At present schemes active in Karnataka are Janani Suraksha Yojana (JSY), Madilu Yojana, Prasuthi Araike Yojana, Thai Bhagya Scheme, Janani Shishu Suraksha Karyakram, Bhagya Lakshmi Yojana, Anganwadi Nutrition Supplementation and 108 Ambulance. These schemes are available for both rural and urban population. Research shows that low proportion of target population receives benefit of such schemes. ${ }^{5,6}$ However, the success of these schemes depends on their utilization by antenatal and postnatal mothers.

Previous studies that have looked at utilization of maternity benefit schemes have mainly focussed on JSY. $^{7,8}$ The present study was undertaken to assess the utilization and their determinants for various maternity benefit schemes among urban slum population of Davangere city.

\section{Objectives}

1. Utilization of maternity benefit schemes among mothers in urban slums of davangere city.

2. Determinants of utilization of these maternity benefit schemes among mothers in urban slums.

\section{METHODS}

It is a community based cross-sectional study conducted from $1^{\text {st }}$ September to $31^{\text {st }}$ November 2015. There are 12 slums under the Urban Health training Centre located in the field practice area of JJM Medical College among them three slums were selected by using simple random sampling. All the mothers who had delivered prior to one year from the start of the study in these slums were recruited for the study. The mothers were identified by door to door enumeration. ANM registers and Anganwadi records were used to identify those mothers who were missed during enumeration. Fifty mothers from each slum were selected using systematic random sampling making a total sample size of 150 .

\section{Inclusion criteria}

1. Postnatal mothers who were residing in the urban slums.

2. Mothers who had delivered prior to one year from start of the study.

3. Mothers who are eligible for maternity benefit schemes as per government criteria.

\section{Exclusion criteria}

1. Mothers not willing to participate and not available at the time of study.

2. Women who had a still birth or abortion

Data collection from these 150 mothers was started after getting institutional ethical review board clearance. Slums were visited with pre intimation and mothers were interviewed using pre-designed, pre-tested, semistructured questionnaire after obtaining informed verbal consent. The questionnaire included information regarding their basic socio-demographic data and factors influencing utilization of maternity benefit schemes.

Data was analysed using SPSS v 17.0 and presented in the form of descriptive statistics (means, proportions, percentages). Chi square test and logistic regression analysis were employed. The strength of association was estimated by calculating the odds ratios (OR) with $95 \%$ confidence intervals (CI). $\mathrm{P}$ value of $<0.05$ was considered statistically significant.

\section{RESULTS}

\section{Socio-demographic characteristics of study participants}

In the present study most of the mothers $(53 \%)$ were in the age group of 20-24 years followed by 25-29 years $(41 \%)$. Mean age of mothers was 21.6 years ( \pm 3.1 years). In our study $39 \%$ of mothers had education up to high school, $36 \%$ up to primary education and $19 \%$ were illiterate. Whereas $33 \%$ of their husbands had education up to primary and $32 \%$ had up to high school and $23 \%$ were illiterate. Majority $(47 \%)$ of the husbands were involved in semi-professional type of occupation and $28 \%$ were involved in skilled occupation. In the present study majority of the mothers, $(77 \%)$ were housewives. In the present study $68(45 \%)$ of the study population belonged to joint family and $48(32 \%)$ to nuclear family. In our study majority, 109 (73\%) of the study population were Hindus. In present study majority $(38 \%)$ of the families belonged to class IV and $29 \%$ belonged to class III according to modified B G Prasad socio-economic classification (Table 1).

Health care workers were the major source of information for mothers (67\%) in our study area. Family and media were the source of information for $17 \%$ and $9 \%$ the mothers respectively (Figure 1).

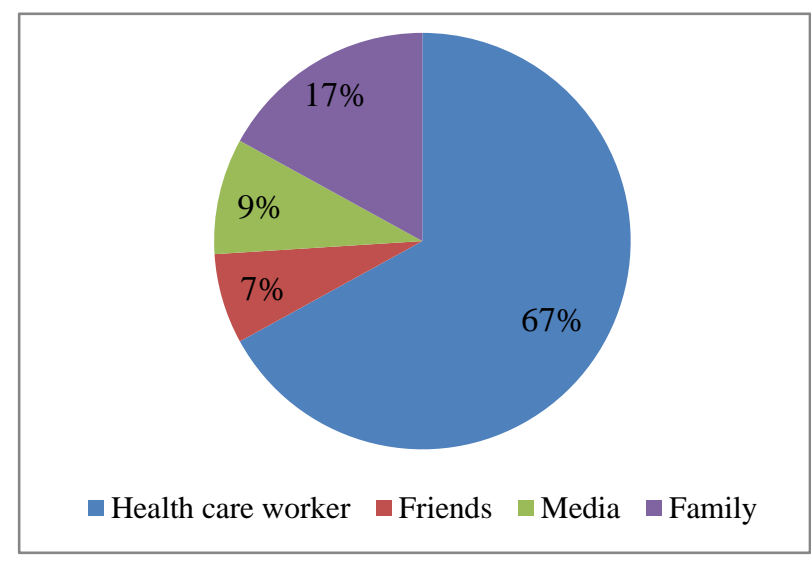

Figure 2: Source of information regarding maternity benefit schemes. 
Table 1: Socio-demographic characteristics of study participants.

\begin{tabular}{|c|c|c|}
\hline Variable & Number & Percentage \\
\hline \multicolumn{3}{|l|}{ Age } \\
\hline$\leq 19$ & 2 & 1 \\
\hline $20-24$ & 79 & 53 \\
\hline $25-29$ & 62 & 41 \\
\hline $30-34$ & 5 & 4 \\
\hline$\geq 35$ & 2 & 1 \\
\hline \multicolumn{3}{|l|}{ Mother's education } \\
\hline Illiterate & 29 & 19 \\
\hline Primary & 53 & 36 \\
\hline High school & 59 & 39 \\
\hline PUC/Diploma & 9 & 6 \\
\hline \multicolumn{3}{|c|}{ Mother's occupation } \\
\hline Home maker & 115 & 77 \\
\hline Unskilled & 10 & 7 \\
\hline Skilled & 10 & 7 \\
\hline Semi-professional & 15 & 9 \\
\hline \multicolumn{3}{|c|}{ Husband's education } \\
\hline Illiterate & 34 & 23 \\
\hline Primary & 49 & 33 \\
\hline High school & 46 & 32 \\
\hline PUC/Diploma & 15 & 9 \\
\hline Degree & 6 & 3 \\
\hline \multicolumn{3}{|c|}{ Husband's occupation } \\
\hline Unskilled & 33 & 22 \\
\hline Semiskilled & 6 & 3 \\
\hline Skilled & 42 & 28 \\
\hline Semi-professional & 69 & 47 \\
\hline \multicolumn{3}{|l|}{ Type of family } \\
\hline Nuclear & 48 & 32 \\
\hline Joint & 68 & 45 \\
\hline Three generation & 34 & 23 \\
\hline \multicolumn{3}{|l|}{ Religion } \\
\hline Hindu & 109 & 73 \\
\hline Muslim & 39 & 26 \\
\hline Others & 2 & 1 \\
\hline \multicolumn{3}{|c|}{ Socio-economic status } \\
\hline I & 3 & 2 \\
\hline II & 14 & 9 \\
\hline III & 43 & 29 \\
\hline IV & 57 & 38 \\
\hline $\mathrm{V}$ & 33 & 22 \\
\hline
\end{tabular}

\section{Utilization of maternity benefit schemes}

In our study majority of the mothers $(68 \%)$ had utilized Anganwadi Nutrition Supplementation and Madilu Yojana followed by JSY and Bhagya Lakshmi Scheme (57\%), Prasuthi Araike Yojana (29\%), JSSK (22\%) and none had utilized Thayi Bhagya Scheme (Table 2).

In our study majority $85(57 \%)$ mothers had utilised Janani Suraksha Yojana, 44 (29\%) mothers had utilized Prasuthi Araike Yojana, $98(65 \%)$ mothers had utilized Madilu Yojana, 102 (68\%) mothers had utilized Anganwadi Nutrition Supplementation, 85 (57\%) mothers had utilized Bhagya Lakshmi Yojana, 84 (56\%) mothers had utilized 108 ambulance and 33 (22\%) mothers had utilized Janani Shishu Suraksha Karyakram. In our study none of mothers had utilized Thayi Bhagya Scheme (Table 2).

Table 2: Utilization of maternity benefit schemes.

\begin{tabular}{|lll|}
\hline $\begin{array}{l}\text { Maternity benefit } \\
\text { schemes }\end{array}$ & $\begin{array}{l}\text { Number of } \\
\text { beneficiaries }\end{array}$ & percentage \\
\hline $\begin{array}{l}\text { Janani Suraksha } \\
\text { Yojana }\end{array}$ & 85 & 57 \\
\hline Prasuthi Araike Yojana & 44 & 29 \\
\hline Madilu Yojana & 98 & 65 \\
\hline $\begin{array}{l}\text { Anganwadi Nutrition } \\
\text { Supplementation }\end{array}$ & 102 & 68 \\
\hline $\begin{array}{l}\text { Bhagya Lakshmi } \\
\text { Yojana }\end{array}$ & 85 & 57 \\
\hline 108 Ambulance & 84 & 56 \\
\hline Thayi bhagya & 0 & 0 \\
\hline $\begin{array}{l}\text { Janani Shishu Suraksha } \\
\text { Karyakram }\end{array}$ & 33 & 22 \\
\hline
\end{tabular}

In present study major reason for non-utilization of maternity benefit schemes was lack of awareness $(80 \%)$ (Table 3).

Table 3: Reasons for non-utilization of maternity benefit schemes.

\begin{tabular}{|lll|}
\hline Reason & Number & Percentage \\
\hline Lack of awareness & 120 & 80 \\
\hline Had to visit several times & 23 & 15 \\
\hline Procedure is complicated & 33 & 22 \\
\hline
\end{tabular}

(Multiple options answered)

\section{Determinants of utilization of maternity benefit schemes among study participants}

In our study utilization of maternity benefit schemes was more among mothers who were literate, housewives, whose husband was literate, belonging to Above Poverty Line (APL) families and joint/three generation family and the association was found to be statistically significant (Table 4).

\section{Multiple logistic regression analysis of utilization of JSY}

Compared with the mothers who were illiterate, those who were literate had better odds of utilizing JSY. Likewise compared to mothers whose husbands were illiterate, mothers whose husbands were literate had more odds of utilising JSY. Similarly compared to nuclear families, odds of utilization of JSY was better among joint/3 generation families. On logistic regression analysis, utilization of JSY was significantly associated with husband's education and type of family (Table 5). 
Table 4: Determinants of utilization of maternity benefit schemes among study participants.

\begin{tabular}{|c|c|c|c|c|c|c|c|}
\hline Variable & $\begin{array}{l}\text { Janani } \\
\text { suraksh } \\
\text { a yojana } \\
\mathbf{N}(\%)\end{array}$ & $\begin{array}{l}\text { Prasuthi } \\
\text { araike } \\
\text { yojana } \\
\text { N }(\%)\end{array}$ & $\begin{array}{l}\text { Madilu } \\
\text { yojana } \\
\text { N }(\%)\end{array}$ & $\begin{array}{l}\text { Anganwadi } \\
\text { nutrition } \\
\text { supplementation } \\
\text { N }(\%)\end{array}$ & $\begin{array}{l}\text { Bhagya } \\
\text { lakshmi } \\
\text { yojana } \\
\text { scheme N }(\%)\end{array}$ & $\begin{array}{l}108 \\
\text { Ambul } \\
\text {-ance } \\
\text { N }(\%)\end{array}$ & $\begin{array}{l}\text { Janani shishu } \\
\text { suraksha } \\
\text { karyakram } \\
\text { N }(\%)\end{array}$ \\
\hline \multicolumn{8}{|l|}{ Age group } \\
\hline$\leq 19$ & $0(0)$ & $0(0)$ & $0(0)$ & $1(1)$ & $1(1)$ & $0(0)$ & $0(0)$ \\
\hline $20-24$ & $45(30)$ & $23(15)$ & $54(36)$ & $55(36)$ & $45(30)$ & $36(24)$ & $21(14)$ \\
\hline $25-29$ & $38(25)$ & $21(14)$ & $42(28)$ & $41(27)$ & $33(22)$ & $23(15)$ & $12(8)$ \\
\hline $30-34$ & $2(1)$ & $0(0)$ & $2(1)$ & $3(2)$ & $5(3)$ & $3(2)$ & $0(0)$ \\
\hline$\geq 35$ & $0(0)$ & $0(0)$ & $98(65)$ & $102(68)$ & $1(1)$ & $2(1)$ & $0(0)$ \\
\hline \multicolumn{8}{|l|}{ Mother's education } \\
\hline Illiterate & $15(10)$ & $9(6)$ & $14(9)$ & $21(14)$ & 19(13) & $10(7)$ & $6(4 \%)$ \\
\hline Primary & $22(15)$ & $17(11)$ & $34(23)$ & $39(26)$ & 30() & $23(15)$ & $17(11)$ \\
\hline High school & $41(27)$ & $16(11)$ & $43(29)$ & $37(25)$ & $30(20)$ & $26(17)$ & $10(7)$ \\
\hline PUC/Diploma & $7(5)$ & $2(1)$ & $7(5)$ & $5(3)$ & $6(4 \%)$ & $5(3)$ & $0(0)$ \\
\hline \multicolumn{8}{|c|}{ Mother's occupation } \\
\hline Home maker & $60(40)$ & $28(19)$ & $68(45)$ & $71(47)$ & $59(39)$ & $56(37)$ & $25(22)$ \\
\hline Unskilled & $7(5)$ & $4(3)$ & $7(5)$ & $10(7)$ & $8(9)$ & $0(0)$ & $3(2)$ \\
\hline Skilled & $8(5)$ & $6(4)$ & $10(7)$ & $9(6)$ & $9(6)$ & $2(1)$ & $1(1)$ \\
\hline Semi-professional & $10(7)$ & $6(4)$ & $13(9)$ & $12(8)$ & $9(6)$ & $6(4)$ & $4(3)$ \\
\hline \multicolumn{8}{|c|}{ Husband's education } \\
\hline Illiterate & $26(17)$ & $15(10)$ & $25(17)$ & $26(17)$ & $23(15)$ & $15(10)$ & $9(6)$ \\
\hline Primary & $25(17)$ & $10(7)$ & $36(24)$ & $33(22)$ & $26(17)$ & $16(11)$ & $5(3)$ \\
\hline High school & $26(17)$ & $14(9)$ & $24(16)$ & $28(18)$ & $24(16)$ & $18(12)$ & $13(9)$ \\
\hline PUC/Diploma & $1(1)$ & $4(3)$ & $8(5)$ & $10(7)$ & $9(6)$ & $13(9)$ & $3(2)$ \\
\hline Degree & $0(0)$ & $0(0)$ & $3(2)$ & $3(2)$ & $2(1)$ & $0(0)$ & $1(1)$ \\
\hline \multicolumn{8}{|c|}{ Husband's occupation } \\
\hline Unskilled & $17(11)$ & $8(5)$ & $21(14)$ & $22(15)$ & $20(13)$ & $10(7)$ & $8(5)$ \\
\hline Semiskilled & $4(3)$ & $1(1)$ & $4(3)$ & $6(4)$ & $4(3)$ & $0(0)$ & $1(1)$ \\
\hline Skilled & $21(14)$ & $9(6)$ & $24(16)$ & $25(17)$ & $27(18)$ & $28(19)$ & $9(6)$ \\
\hline Semi-professional & $43(29)$ & $26(17)$ & $49(33)$ & $49(33)$ & $34(23)$ & $26(17)$ & $15(10)$ \\
\hline \multicolumn{8}{|l|}{ Type of family } \\
\hline Nuclear & $20(13)$ & $8(5)$ & $26(17)$ & $32(21)$ & $26(17)$ & $25(17)$ & $11(7)$ \\
\hline Joint & $42(28)$ & $31(21)$ & $52(35)$ & $54(36)$ & $44(29)$ & 21(14) & $14(9)$ \\
\hline Three generation & $23(15)$ & $5(3)$ & $20(13)$ & $16(11)$ & $15(10)$ & $18(12)$ & $8(5)$ \\
\hline \multicolumn{8}{|c|}{ Socio-economic status } \\
\hline I & $3(2)$ & $0(0)$ & $3(2)$ & $1(1)$ & $1(1)$ & $3(2)$ & $0(0)$ \\
\hline II & $6(4)$ & $8(5)$ & $11(7)$ & $11(7)$ & $7(5)$ & $7(5)$ & $5(3)$ \\
\hline III & $31(21)$ & $18(12)$ & $26(17)$ & $34(23)$ & $28(19)$ & $11(7)$ & $12(8)$ \\
\hline IV & 28(19) & $15(10)$ & $33(22)$ & $40(27)$ & $33(22)$ & $24(16)$ & $13(9)$ \\
\hline V & $17(11)$ & $3(2)$ & $25(17)$ & $16(11)$ & $16(11)$ & 19(13) & $3(2)$ \\
\hline
\end{tabular}

\section{Multiple logistic regression analysis of utilization of Prasuthi Araike Yojana}

Compared to nuclear families, odds of utilization of Prasuthi Araike Yojana was better among joint $/ 3$ Generation families. Likewise compared to Below Poverty line Families (BPL), odds of utilization of Prasuthi Araike Yojana was better among Above Poverty line Families (APL). On logistic regression analysis, utilization of Prasuthi Araike Yojana was significantly associated with type of family and socioeconomic status (Table 6).

\section{Multiple logistic regression analysis of utilization of Madilu Yojana}

Compared to working mothers, odds of utilization of Madilu Yojana was better among housewives. Likewise compared to nuclear families, odds of utilization of Madilu Yojana was better among joint/3G families. On logistic regression analysis, utilization of Madilu Yojana 
was significantly associated with mother's occupation and type of family (Table 7).

Table 5: Multiple logistic regression analysis of utilization of JSY.

\begin{tabular}{|c|c|c|c|c|}
\hline \multirow{2}{*}{ Variable } & \multirow{2}{*}{$\begin{array}{l}\text { Odds } \\
\text { ratio }\end{array}$} & \multicolumn{2}{|c|}{$\begin{array}{l}\text { Confidence } \\
\text { interval }\end{array}$} & \multirow{2}{*}{$\begin{array}{l}P \\
\text { value }\end{array}$} \\
\hline & & $\begin{array}{l}\text { Upper } \\
\text { limit }\end{array}$ & $\begin{array}{l}\text { Lower } \\
\text { limit }\end{array}$ & \\
\hline $\begin{array}{l}\text { Mothers } \\
\text { education } \\
\text { literate illiterate }\end{array}$ & 0.73 & 0.33 & 1.62 & 0.438 \\
\hline $\begin{array}{l}\text { Husbands } \\
\text { education literate } \\
\text { illiterate }^{\neq}\end{array}$ & 3.13 & 1.31 & 7.50 & 0.01 \\
\hline $\begin{array}{l}\text { Type of family } \\
\text { joint } / 3 \text { generation } \\
\text { nuclear }^{\neq}\end{array}$ & 0.40 & 0.20 & 0.82 & 0.01 \\
\hline
\end{tabular}

Table 6: Multiple logistic regression analysis of utilization of Prasuthi Araike Yojana.

\begin{tabular}{|c|c|c|c|c|}
\hline \multirow{2}{*}{ Variable } & \multirow{2}{*}{$\begin{array}{l}\text { Odds } \\
\text { ratio }\end{array}$} & \multicolumn{2}{|c|}{$\begin{array}{l}\text { Confidence } \\
\text { interval }\end{array}$} & \multirow{2}{*}{$\begin{array}{l}P \\
\text { value }\end{array}$} \\
\hline & & $\begin{array}{l}\text { Upper } \\
\text { limit }\end{array}$ & $\begin{array}{l}\text { Lower } \\
\text { limit }\end{array}$ & \\
\hline $\begin{array}{l}\text { Type of family } \\
\text { joint } / 3 \text { generation } \\
\text { nuclear }^{\neq}\end{array}$ & 0.36 & 0.15 & 0.86 & 0.02 \\
\hline $\begin{array}{l}\text { Socioeconomic } \\
\text { status } \\
\text { APL } \\
\text { BPL }^{\neq}\end{array}$ & 0.32 & 0.15 & 0.67 & 0.02 \\
\hline
\end{tabular}

\# Reference group

Table 7: Multiple logistic regression analysis of utilization of Madilu Yojana.

\begin{tabular}{|c|c|c|c|c|}
\hline \multirow{2}{*}{ Variable } & \multirow{2}{*}{$\begin{array}{l}\text { Odds } \\
\text { ratio }\end{array}$} & \multicolumn{2}{|c|}{$\begin{array}{l}\text { Confidence } \\
\text { interval }\end{array}$} & \multirow{2}{*}{$\begin{array}{l}\mathbf{P} \\
\text { value }\end{array}$} \\
\hline & & $\begin{array}{l}\text { Upper } \\
\text { limit }\end{array}$ & $\begin{array}{l}\text { Lower } \\
\text { limit }\end{array}$ & \\
\hline $\begin{array}{l}\text { Mothers } \\
\text { occupation } \\
\text { not working } \\
\text { working }^{\neq}\end{array}$ & 4.14 & 1.5 & 11.46 & 0.006 \\
\hline $\begin{array}{l}\text { Type of family } \\
\text { joint } / 3 \text { generation } \\
\text { nuclear }^{\neq}\end{array}$ & 0.52 & 0.25 & 1.06 & 0.07 \\
\hline
\end{tabular}

Multiple logistic regression analysis of utilization of Anganwadi Nutrition Supplementation

Compared to working mothers, odds of utilization of Anganwadi Nutrition Supplementation was better among housewives. Likewise compared to nuclear families, odds of utilization of Madilu Yojana was better among joint/3G families. Similarly compare to Below Poverty line Families (BPL), odds of utilization of Anganwadi Nutrition Supplementation was better among Above Poverty line Families (APL). On logistic regression analysis, utilization of Anganwadi Nutrition Supplementation was significantly associated with mother's occupation, type of family and socioeconomic status (Table 8).

Table 8: Multiple logistic regression analysis of utilization of Anganwadi Nutrition Supplementation.

\begin{tabular}{|c|c|c|c|c|}
\hline \multirow{2}{*}{ Variable } & \multirow{2}{*}{$\begin{array}{l}\text { Odds } \\
\text { ratio }\end{array}$} & \multicolumn{2}{|c|}{$\begin{array}{l}\text { Confidence } \\
\text { interval }\end{array}$} & \multirow{2}{*}{$\begin{array}{l}P \\
\text { value }\end{array}$} \\
\hline & & $\begin{array}{l}\text { Upper } \\
\text { limit }\end{array}$ & $\begin{array}{l}\text { Lower } \\
\text { limit }\end{array}$ & \\
\hline $\begin{array}{l}\text { Mothers } \\
\text { occupation } \\
\text { not working } \\
\text { working }^{\neq}\end{array}$ & 4.80 & 1.58 & 14.53 & 0.005 \\
\hline $\begin{array}{l}\text { Type of family } \\
\text { joint } / 3 \text { generation } \\
\text { nuclear }^{\neq}\end{array}$ & 0.91 & 0.43 & 1.90 & 0.81 \\
\hline $\begin{array}{l}\text { Socioeconomic } \\
\text { status } \\
\text { APL } \\
\text { BPL }^{\neq}\end{array}$ & 0.50 & 0.24 & 1.04 & 0.06 \\
\hline
\end{tabular}

Table 9: Multiple logistic regression analysis of utilization of 108 ambulance.

\begin{tabular}{|c|c|c|c|c|}
\hline \multirow{2}{*}{ Variable } & \multirow{2}{*}{$\begin{array}{l}\text { Odds } \\
\text { ratio }\end{array}$} & \multicolumn{2}{|c|}{$\begin{array}{l}\text { Confidence } \\
\text { interval }\end{array}$} & \multirow{2}{*}{$\begin{array}{l}P \\
\text { value }\end{array}$} \\
\hline & & $\begin{array}{l}\text { Upper } \\
\text { limit }\end{array}$ & $\begin{array}{l}\text { Lower } \\
\text { limit }\end{array}$ & \\
\hline $\begin{array}{l}\text { Mothers } \\
\text { occupation } \\
\text { Not working } \\
\text { working }^{\neq}\end{array}$ & 0.31 & 0.13 & 0.74 & 0.008 \\
\hline $\begin{array}{l}\text { Husbands } \\
\text { education } \\
\text { Literate illiterate }\end{array}$ & 1.07 & 0.49 & 2.33 & 0.84 \\
\hline $\begin{array}{l}\text { Type of family } \\
\text { joint } / 3 \mathrm{G} \\
\text { nuclear }^{\neq}\end{array}$ & 1.75 & 0.87 & 3.5 & 0.01 \\
\hline $\begin{array}{l}\text { Socioeconomic } \\
\text { status } \\
\text { APL } \\
\text { BPL }^{\neq}\end{array}$ & 0.58 & 0.30 & 1.15 & 0.12 \\
\hline
\end{tabular}

Multiple logistic regression analysis of utilization of 108 Ambulance

Compared to working mothers, odds of utilization of 108 Ambulance was better among housewives. Likewise compared to nuclear families, odds of utilization of 108 
Ambulance was better among joint/3G families. Similarly compare to Below Poverty line Families (BPL), odds of utilization of 108 Ambulance was better among Above Poverty line Families (APL). Similarly compared to mothers whose husbands were illiterate, mothers whose husbands were literate had more odds of utilising 108 Ambulance. On logistic regression analysis, utilization of 108 Ambulance was significantly associated with mother's occupation (Table 9).

\section{DISCUSSION}

In our study $57 \%$ of mothers had utilized Janani Suraksha Yojana and almost similar findings were observed by Vikas Kumar, et al (53.4\%). ${ }^{7}$ Our study result is higher than study conducted by Harpreet Kaur, et al (48.2\%), Vikram K, et al (27.3\%), and lower than study by Raut M $\mathrm{M}$, et al $(80.3 \%){ }^{8-10}$

In present study $68 \%$ of mothers had utilized Anganwadi Nutrition Supplementation which is lower than study by Jawahar Preethy, et al (74.1\%). ${ }^{11}$ In present study $57 \%$ of mothers had utilized Bhagyalakshmi Yojana and almost similar findings were observed by Minu Joseph, et al $(54.2 \%){ }^{12}$ In present study $56 \%$ of mothers had utilized 108 ambulance which is higher than study by Hitesh Bhabhor, et al $(39 \%){ }^{13}$ In our study $22 \%$ of mothers utilized Janani Shishu Suraksha Karyakram and almost similar findings were observed by R. C. Goyal, et al $(20 \%) .{ }^{14}$ But our study result is lower than study by Uvi Tyagi, et al $(60 \%){ }^{15}$

In present study major reason for non-utilization of schemes is lack of awareness and similar finding was observed by Sahu D, et al. ${ }^{16}$

\section{CONCLUSION}

Overall the utilization of various maternity benefit schemes is poor among beneficiaries. The major source of information regarding these schemes are health care workers, despite of this major reason for non -utilization of schemes is lack of awareness. Mother's and their husband's literacy status, occupation of mother, type of family, socioeconomic status are the important factors that affect utilization of various schemes.

\section{Recommendations}

- There is need to create awareness and counsel the mothers, family members and community as a whole for better utilization of various maternity benefit schemes.

- The procedure of schemes needs to be simplified for maximum utilization of schemes.

- For complete utilization of maternity benefit schemes it is important to pay attention to different socioeconomic factors.

\section{ACKNOWLEDGMENTS}

I am thankful to all mothers who were part of the study for their kind co-operation. I also thank my colleagues, post graduate students for their support during the course of the study.

Funding: No funding sources Conflict of interest: None declared

Ethical approval: The study was approved by the Institutional Ethics Committee

\section{REFERENCES}

1. Singh PK, Rai RK, Alagarajan M, Singh L. Determinants of Maternity Care Services Utilization among Married Adolescents in Rural India. PLoS ONE. 2012;7(2):1-14.

2. Maternal mortality. Fact sheet number 348. Available from URL www.http://www.who.int/ mediacentre/ factsheets/. Accessed on 12th November 2015.

3. National health mission, ministry of health and family welfare, government of India. http://nrhm. gov.in/images/nrhm-logo.png. Accessed on.

4. Rise in number of institutional child delivery. Press information bureau, Government of India, Ministry of Health and Family Welfare. Available from URL http://pib.nic.in/newsite/PrintRelease.aspx?relid=12 3989. Accessed on 12th November 2015.

5. Johnson AR, Rock B, Catherin N, Berlin, Rupini R, Kasthuri A. Awareness of Government Maternity Benefit Schemes among women attending antenatal clinic in a rural hospital in Karnataka, India. International journal of current research and academic review. 2015;3(1):137-43.

6. Vora K, Yasobant S, Mavalankar D. Predictors of Availing Maternal Health Schemes: A community based study in Gujarat, India. Indian Journal of Community Health. 2014;26(2):174-8.

7. Kumar V, Misra SK, Kaushal SK, Gupta SC, Maroof AK. Yojana JS. Its utilization and perception among mothers and health care providers in a rural area of North India. International journal of medicine and public health. 2015;5(2):165-68.

8. Kaur H, Kaur A, Kaur H, Devgun P. A study of utilization of Janani Suraksha Yojana (JSY) scheme, among beneficiaries in a rural area of Punjab. National Journal of Research in Community Medicine. 2015;4(1):114-23.

9. Vikram K, Sharma AK, Kannan AT. Beneficiary level factors influencing Janani Suraksha Yojana utilization in urban slum population of transYamuna area of Delhi.the indian journal of medical research. 2013;138(3):340-6.

10. Raut MM, Malkar VR, Joge US, Choudhari SG. An exploratory study on knowledge and utilization pattern of Janani Suraksha Yojana (JSY) among beneficiaries in Akola district of Maharashtra state. 
Health and Population - Perspectives and Issues. 2011;34(4);204-14.

11. Preethy J, Ansuya NM. Astudy to identify the knowledge and utilization of Integrated Child Development Schemes (ICDS) services among women in Udupi District, Karnataka. International Journl of Nursing Education. 2011;3(2):79-82.

12. Joseph M, Manjula A. Assessment of knowledge and utilization of Below Poverty Line (BPL) Schemes among BPL families. Journal of Nursing and Health Science. 2014;3(6):1-5.

13. Bhabhor H, Chhaya J, Machhar U, Devalia J, Talsania N. A Cross-Sectional Study on Utilization of 108 EMRI Obstetric Care Services for Institutional Delivery in Gandhinagar District of Gujarat. Journal of Dental and Medical Sciences. 2015;14(9):36-40.

14. Goyal RC, Singh PL, Mudey A. Assessment of implementation status of Janani-Shishu Suraksha Karyakram (JSSK) for free referral transport services at selected Public health facilities in Wardha district, of Central India. International Journal of Current Research and Review. 2014;6(16):29-34.

15. Tyagi U, Pattabi K, Kaur P. Utilization of services under Janani Shishu Suraksha Karyakram for institutional deliveries in the public sector facilities, Sirmaur District, Himachal Pradesh, India. Indian Journal of Community Medicine. 2016;41(1).

16. Sahu D, Gandhi NK, Soni GP. Knowledge and utilization of Janani Suraksha Yojana (JSY): An epidemiological study. Indian Journal of Maternal and Child Health. 2012;14(1):7.

Cite this article as: Angadi N, Davalgi S,

Raghavendra SK. Determinants of utilization of maternity benefit schemes among mothers in urban slums of Davangere city, Karnataka, India. Int J Community Med Public Health 2016;3:651-7. 